\title{
Leaked internal report criticizes government oversight of antimicrobial resistance
}

$\mathrm{T}$

he federal government's oversight of antimicrobial resistance is sorely plagued by deficiencies, according to a draft report prepared by officials at the Public Health Agency of Canada (PHAC).

The internal government report, Antimicrobial Resistance in Canada: A Gap Analysis, argues that the government needs to tackle a series of "key" deficiencies in its current efforts to address antimicrobial resistance (AMR).

Those include the:

- "absence of a national AMR surveillance system or plan;

- absence of an AMR governance structure or action plan;

- absence of a governance structure for antimicrobial stewardship; [and]

- absence of coherent federal AMR policy."

The report, currently being circulated amongst members of an antimicrobial resistance task force, describes antimicrobial resistance as a "growing problem" that will undermine "government efforts to improve quality of health care, to increase access and to decrease wait times." In support of that proposition, the report estimates that antibiotic-resistant health care-associated infections are associated with a nearly $30 \%$ increase in hospital costs. "Delayed or inappropriate therapy for infections caused by resistant organisms may result in longer hospital stays and a need for additional care. ... Limited treatment options may also add to the burden as more expensive and/or toxic therapies may be required."

The report's recommendations closely match advice given to PHAC by the now-disbanded Canadian Committee on Antibiotic Resistance in 2009 (www.cmaj.ca/lookup/doi/10.1503/cmaj .109-3109).

The World Health Organization has called on Canada and other member nations to estimate the costs of all elements of a national antimicrobial resistance plan, "develop a complete budget,

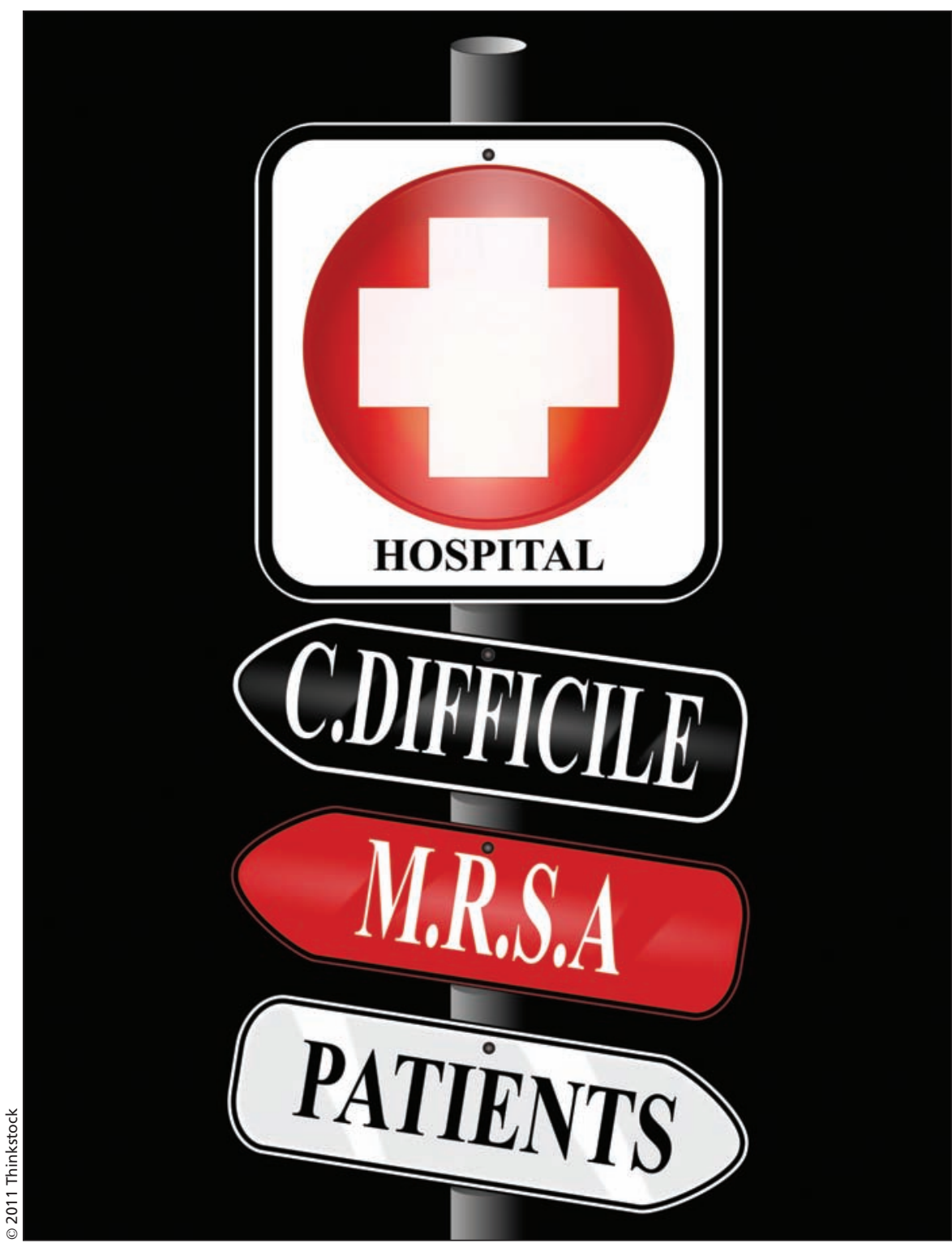

Antibiotic resistant health care-associated infections are associated with a nearly $30 \%$ increase in hospital costs, according to a leaked government report.

and integrate it into the national health budget and other relevant budgets" (www.who.int/world-health-day/2011 /presskit/WHDIntrototobriefs.pdf).

PHAC officials note in the report that Canada lags behind Australia, the United States, the United Kingdom and other countries in Europe in developing antibiotic use guidelines.
As well, "existing surveillance systems only capture some of the data identified as critical for comprehensive AMR surveillance," the report states. "Limited data are available regarding antimicrobial usage in non-hospital settings, notably in the community and in the agri-food sector." It also notes that the use of human drugs in meat 
production - both to treat illness and "for non-therapeutic purposes, such as growth promotion" - has become a serious concern. "This may lead to the transfer of antimicrobial-resistant pathogens, such as Salmonella, Campylobacter and Escherichia coli, to humans via direct contact with animals, or through the consumption of contaminated food or water."

The report recommends that the government develop an effective AMR surveillance system, as well as "introduce legal requirements for manufacturers to collect and report data on antimicrobial distribution [and] develop stronger auditing procedures from importer to end-user, and [a] stronger monitoring and audit system of antibiotic usage in all areas (medical, veterinary, etc.)."

PHAC spokeswoman Charlene Wiles indicated in a written statement that the agency hopes to use the analysis as the basis for "a coherent path forward to address antimicrobial resistance in Canada."

Wiles added that discussions are underway between PHAC and provincial/territorial public health officials on antimicrobial resistance matters including "deciding which additional diseases/ conditions of common interest related to AMR should be the focus of national surveillance, with the understanding that each of the provinces and territories will need to subsequently establish the necessary mechanisms (e.g. mandatory reporting) within their jurisdictions in order to obtain valid data as part of a comprehensive national surveillance effort." But the notion that responsibility for antimicrobial resistance management would be partially downloaded onto provincial and territorial government was criticized by Melissa Coleman, an Ottawa-based public health researcher. She argues that the international and interjurisdictional nature of AMR problems warrants strong federal leadership and investment.

Dr. Margaret Fast, scientific director of the National Collaborating Centre for Infectious Diseases, says there's a need to fill the current leadership "void" on the file. Fast says the centre is now serving as a "placeholder" to prevent antimicrobial efforts from col- lapsing while the government deliberates future plans.

"There is a lack of clarity on how this happens," Fast says. "Where does the money come from? Where does the leadership come from? It certainly is taking time to develop a framework. What we need is clarity about what the federal government is willing and able to take on."

Dr. Lynora Saxinger, chair of the antimicrobial stewardship and resistance committee for the Association of Medical Microbiology and Infectious Disease Canada, and associate professor in the Department of Medical Microbiology and Immunology at the University of Alberta in Edmonton, argues that the time is ripe for a "national action plan and a governance structure."

Saxinger says the government should adopt a straight-line resolution to the antimicrobial resistance problem. "The protocols used by other countries could be easily adopted. But bold leadership is now required." - Paul Christopher Webster, Toronto, Ont.

CMAJ 2011. DOI:10.1503/cmaj.109-3921 